\title{
5G and LTE-TDD Synchronized Coexistence with Blind Retransmission and Mini-Slot Uplink
}

\author{
Abdelrahim Mohamed, Atta Quddus, Pei Xiao, Bernard Hunt, Rahim Tafazolli \\ Institute for Communications Systems (ICS), Home of 5GIC, University of Surrey, Guildford, UK \\ E-mail: \{abdelrahim.mohamed; a.quddus; p.xiao; b.hunt; r.tafazolli @ surrey.ac.uk\}
}

\begin{abstract}
The fifth-generation (5G) new radio (NR) cellular system promises a significant increase in capacity with reduced latency. However, the 5G NR system will be deployed along with legacy cellular systems such as the long-term evolution (LTE). Scarcity of spectrum resources in low frequency bands motivates adjacent-/co-carrier deployments. This approach comes with a wide range of practical benefits and it improves spectrum utilization by re-using the LTE bands. However, such deployments restrict the 5G NR flexibility in terms of frame allocations to avoid the most critical mutual adjacent-channel interference. This in turns prevents achieving the promised 5G NR latency figures. In this we paper, we tackle this issue by proposing to use the minislot uplink feature of 5G NR to perform uplink acknowledgment and feedback to reduce the frame latency with selective blind retransmission to overcome the effect of interference. Extensive system-level simulations under realistic scenarios show that the proposed solution can reduce the peak frame latency for feedback and acknowledgment up to $33 \%$ and for retransmission by up to $25 \%$ at a marginal cost of an up to $3 \%$ reduction in throughput.
\end{abstract}

Index Terms-5G new radio; frame structure; latency; longterm evolution; time division duplex.

\section{INTRODUCTION}

Recently, the fifth-generation $(5 \mathrm{G})$ new radio (NR) has been standardised by the Third Generation Partnership Project (3GPP). The new cellular system supports diverse applications/verticals under three main use cases. These include: enhanced mobile broadband (eMBB), massive machine-type communication (mMTC), and ultra-reliable and low latency communication (URLLC). On the radio access network (RAN) side, the 5G NR has been designed in a flexible and reconfigurable manner to meet the performance targets of $4 \mathrm{~ms}$ and 1 ms user plane latency for eMBB and URLLC respectively, 20 Gbps peak data rate, and $100 \mathrm{Mbps}$ user experienced data rate [1]. Most of these targets/features are linked to the frame structure. Consequently, the 3GPP specified a flexible 5G NR frame supporting different numerologies with several subcarrier spacings along with uplink/downlink switching at the symbol-level rather than the slot-level switching adopted in the long-term evolution (LTE). Such features contribute towards achieving the 5G NR targets, in particular the latency requirements, whilst adapting the transmission direction according to the actual traffic demand.

The 5G NR will be deployed with the existing 4G (i.e., the LTE) system. In fact, the non-standalone 5G NR (in initial rollout) will use the LTE as an anchor point in a dual connectivity configuration [2], [3] whereas the standalone $5 \mathrm{G}$ NR will be independent of the LTE system [4]. In both cases, the 5G NR and the LTE will be operating in the same area. Due to the limited spectrum availability in sub$6 \mathrm{GHz}$ bands, adjacent channel deployment will be required [5]. However, such deployments result in interference between the $5 \mathrm{G} \mathrm{NR}$ and the $4 \mathrm{G}$ networks due to spectral leakage from the interfering nodes which can lead to desensitisation in the victim's receiver. In addition, the adjacent deployment of $4 \mathrm{G}$ and $5 \mathrm{G}$ NR systems can result in blocking the victim's receiver and overloading the radio frequency front-end. Such interference could be generated from the LTE base stations (BSs), known as e-NodeBs, and the LTE user equipment (UE) towards the $5 \mathrm{G}$ BSs, known as g-NodeBs, and the $5 \mathrm{G} \mathrm{UE}$, and vice versa. The European Communication Committee (ECC) defines strict out-of-block power limits to solve this issue. However, current systems cannot achieve these limits costeffectively [6]. To overcome this challenge, the ECC proposes synchronized deployment with fully aligned frames [6]. Nevertheless, this solution comes at the expense of increased $5 \mathrm{G}$ NR frame latency beyond the $4 \mathrm{~ms}$ target as well as reduced flexibility for uplink/downlink switching.

In this paper, we propose a solution to this problem by utilizing a mini-slot uplink with selective blind retransmissions for 5G NR signaling. The proposed solution maintains the frame alignment and reduces the $5 \mathrm{G}$ NR frame latency to contribute towards meeting the $5 \mathrm{G}$ NR latency targets. To overcome the effect of spectral leakage from the LTE e-NodeBs, we propose blind retransmission for some of the 5G UE whose mini-slot uplink is lost due to the adjacent channel interference. It is worth mentioning that the concept of mini-slot is not new and it was previously discussed and proposed for 5G. On top of this concept, we contribute by proposing blind retransmission to overcome the effect of additional interference in the minislot uplink from LTE BSs along with providing results from detailed system-level simulations in 3GPP and ECC scenarios. The reminder of this paper is structured as follows. Section II discusses details of the problem and presents the proposed solution. Section III provides and discusses system-level simulation results in realistic scenarios. Finally, conclusions are drawn in Section IV.

\section{Coexistence with Mini-Slot Uplink}

\section{A. 4G/5G Coexistence Issues}

Performance targets and use cases of the 5G NR system motivated the $3 \mathrm{GPP}$ to specify two frequency ranges for $5 \mathrm{G}$ operation: $0.41-7.125 \mathrm{GHz}$ (known as sub-6 $\mathrm{GHz}$ or frequency range 1), and $24.25-52.6 \mathrm{GHz}$ (known as millimetrewave or frequency range 2) [7]. In the sub-6 GHz range, parts of the $5 \mathrm{G}$ NR bands are also being used for LTE. For example, the $3.4-3.8 \mathrm{GHz}$ band is being considered as the primary $5 \mathrm{G}$ NR band in Europe and it is also being used for LTE, with both systems operating in time division duplex (TDD) mode. In the latter, deploying the 5G NR adjacent to the LTE system could result in four types of interference as shown in Fig. 1. These include:

- Interference between LTE e-NodeB and 5G g-NodeB: When one of the networks is operating in downlink 


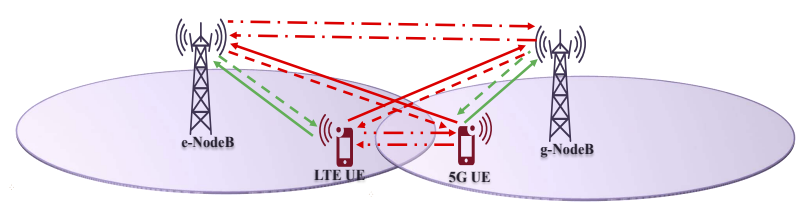

$\longrightarrow$ Uplink signal

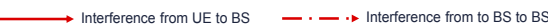

$\ldots$ - - $\rightarrow$ Downlink signal $\quad \ldots \ldots$ Interference from BS to UE $\quad \ldots \rightarrow$ Interference from UE to UE

Figure 1: Signal and interference paths between $4 \mathrm{G}$ and $5 \mathrm{G}$.

(interferer) and the other network is operating in the uplink (interfered).

- Interference between LTE e-NodeB and 5G UE: When both networks are operating in downlink (LTE e-NodeB is the interferer) or in uplink (5G UE is the interferer).

- Interference between LTE UE and 5G g-NodeB: When both networks are operating in downlink (5G g-NodeB is the interferer) or in uplink (LTE UE is the interferer).

- Interference between LTE UE and 5G UE: When one of the networks is operating in uplink (interferer) and the other network is operating in the downlink (interfered).

Intermittent activity and the low power of mobile devices [8] reduces criticality of the interference between LTE UE and 5G UE. In addition, the UE-BS interference can be handled by traditional interference management techniques such as almost-blank subframes or enhanced inter-cell interference coordination. Since the BSs tend to have fixed locations and they operate continuously, the interference between the LTE e-NodeB and the $5 \mathrm{G}$ g-NodeB is considered as the most critical and it is regulated. Several studies have shown that either very expensive non-commercial filters or large guard bands are needed to reduce the effect of this interference [6]. Consequently, a synchronized operation with fully aligned frames or a tight coordination with common scheduling are considered as the most effective solutions.

In the aligned frames approach, both networks (i.e., the $5 \mathrm{G}$ NR and the LTE) use the same uplink/downlink allocations and switching ratios such that when one of the networks is operating in downlink (or uplink) the other network will also be operating in downlink (or uplink). This in turns avoids the interference between the LTE e-NodeB and the $5 \mathrm{G}$ g-NodeB. A similar problem exists in dynamic TDD that has been proposed for LTE-B. The latter shares all the time slots between the uplink and the downlink with flexible slot reconfiguration. To avoid the BS-to-BS interference, the authors of [9] propose a hybrid frequency division duplex (FDD) and TDD coordination scheme by scheduling the uplink and the downlink for each user in different carriers and at different subframes. This solution can be adopted when all BSs are controlled by the same operator since it requires a common scheduler. Consequently, it may not be applicable to $4 \mathrm{G} / 5 \mathrm{G}$ coexistence since the networks can be controlled by different operators with independent schedulers.

The asynchronous operation (i.e., when the $5 \mathrm{G}$ and LTE frames are not aligned) has been investigated by the ECC. The results reported in [6] and the references therein indicate that if the frames are not aligned, then large separation distances up to $60 \mathrm{~km}$ may be needed to ensure non-harmful interference. This is based on the fact that the current $5 \mathrm{G}$ technology cannot cost-effectively achieve the restricted power limit specified by regulatory bodies for asynchronous systems. In this direction,
Frame $=10$ Subframes $=10 \mathrm{~ms}$ (Fixed)

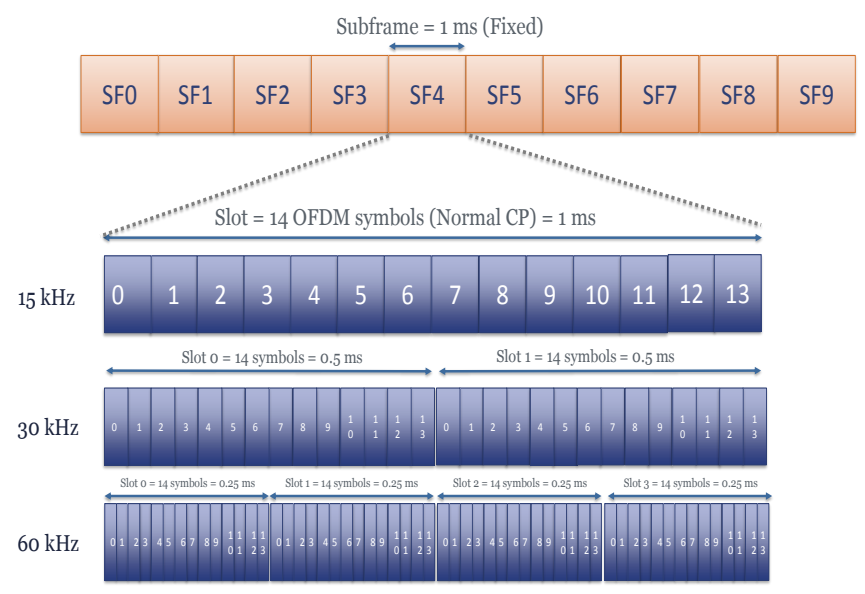

Figure 2: 5G New Radio frame, subframe and slot format. Acronyms SF: Subframe, CP: Cyclic prefix.

the ECC showed benefits of using the asynchronous system for $5 \mathrm{G}$ (in particular the flexibility for uplink/downlink allocations and latency), however, they clearly indicated that such asynchronous operation may not be feasible due to the very large separation distance required. This resulted in suggesting the synchronous operation (i.e., both frames are aligned) as a feasible solution to allow coexistence of both systems in the same band. Ofcom, the U.K. spectrum regulator, followed a similar approach and suggested the synchronized operation in the considered band. To avoid repeating the work already done in standardization and regulatory bodies, we consider the synchronous system and provide a solution to tackle the main drawback of this operation (i.e., the high latency).

Given the constraints mentioned above, we consider the aligned frames approach. Similar to the LTE, the 5G NR radio frame duration is fixed at $10 \mathrm{~ms}$ and it consists of 10 subframes. Depending on the subcarrier spacing, the subframe consists of one or multiple time slots, with the latter consisting of 14 symbols and can contain all downlink, all uplink, or at least one downlink part and at least one uplink part. Fig. 2 shows the generic $5 \mathrm{G} \mathrm{NR}$ frame and slot format. It can be noticed that the 5G NR frame is aligned with the LTE frame at the sub-frame and at the frame boundaries, and complete alignment at the symbol level of uplink/downlink transmissions between the LTE-TDD and the 5G NR can be achieved. Such alignment becomes critical when the LTE-TDD and the 5G NR are deployed in adjacent channels. However, several of the $5 \mathrm{G}$ NR benefits are linked to the frame structure, and restricting the $5 \mathrm{G} \mathrm{NR}$ frame to be fully aligned with the LTE-TDD frame in terms of restricted uplink/downlink symbols would have implications on the minimum latency achievable by the $5 \mathrm{G}$ NR.

Considering the $3.4-3.8 \mathrm{GHz}$ band, the ECC identified LTE-TDD frame configuration 2 with special subframe 7 is fully aligned with the 5G NR frame (DDDDDDDSUU) with $30 \mathrm{kHz}$ subcarrier spacing, as shown in Fig. 3. This frame format avoids the interference between the LTE e-NodeB and the 5G g-NodeB. Consequently, it allows adjacent networks to coexist without large guard bands or additional filters. However, this will result in increased 5G NR latency and it does not allow optimizing the uplink/downlink ratio. In fact, 


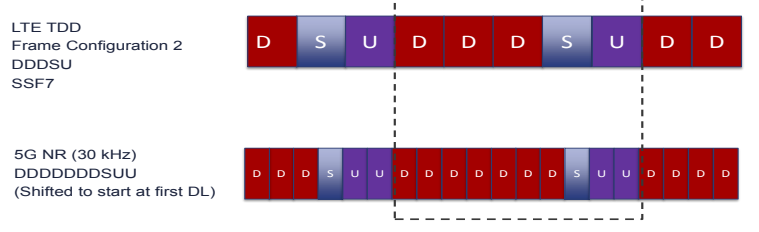

Figure 3: LTE-TDD Frame configuration 2 and 5G NR Frame (DDDDDDDSUU) with $30 \mathrm{kHz}$ subcarrier spacing. Acronyms D: Downlink, U: Uplink, S: Special.

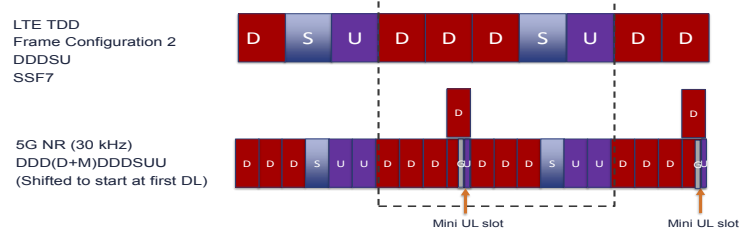

Figure 4: LTE-TDD Frame configuration 2 and 5G NR Frame with $30 \mathrm{kHz}$ subcarrier spacing and mini-uplink slot.

the $5 \mathrm{G}$ NR frame in Fig. 3 will result in a maximum layer 1 latency $>4 \mathrm{~ms}$ [6], which violates the eMBB $4 \mathrm{~ms}$ latency target when adding all higher layer processing latencies. In the following, we propose a scheme to solve this problem partially by reducing the $5 \mathrm{G}$ NR frame latency for feedback and retransmission whilst maintaining the alignment benefits.

\section{B. Mini-Slot Uplink with Selective Blind Retransmission}

To overcome the 5G NR frame latency issue whilst maintaining alignment with the LTE frame, we propose utilizing the mini-slot uplink feature of the 5G NR standard to reduce the latency and to offer finer granularity for scheduling [10]. We consider a mini-slot consisting of two uplink symbols along with a guard period. Fig. 4 shows an example of the $5 \mathrm{G}$ NR frame with the mini-slot uplink. This approach allows more frequent uplink feedback, thus reducing the $5 \mathrm{G} \mathrm{NR}$ frame latency. Considering a generic 5G NR frame with $N_{d}$ consecutive downlink slots violating a maximum frame latency threshold of $L_{t h r}$, the number of required mini-slot uplinks $N_{m u}$ can be formulated as:

$$
N_{m u}=\left\{\begin{array}{cl}
0 & , \text { for } \quad N_{d} \cdot T_{s} \leq L_{t h r} \\
\left\lceil\frac{N_{d} \cdot T_{s}}{L_{t h r}}\right\rceil & , \text { for } \quad N_{d} \cdot T_{s}>L_{t h r}
\end{array}\right\},
$$

where $\lceil x\rceil$ is the ceiling operator, i.e., the smallest integer greater than or equal to $x, T_{s}$ is the slot duration which can be obtained as

$$
T_{s}=K\left(\frac{1}{\Delta_{f}}+T_{c}\right)
$$

where $K$ is the number of symbols per slot, $\Delta_{f}$ is the subcarrier spacing, and $T_{c}$ is the cyclic prefix duration. The optimal location for the mini-slots can be found by dividing $N_{d}$ into $N_{m u}$ regions and placing a mini-slot in the middle of each region, i.e.,

$$
C_{i}=\left\lceil\frac{N_{d}}{N_{m u}}(i-0.5)\right\rceil, i=1,2,3, \ldots, N_{m u}
$$

where $C_{i}$ is the location of mini-slot $i$ referenced to the start of the block containing consecutive downlink slots.

Switching the transmission direction between the downlink and the mini-slot uplink requires a guard period $T_{g}$ to cope with hardware switching time $T_{h}$, channel delay spread $\sigma_{\tau}$, propagation delay $T_{p}$ as well as the filter response time $T_{f}$ [11], [12]. The lower bound for the guard period can be formulated as [12], [13]:

$$
T_{g}=T_{h}+\sigma_{\tau}+T_{p}+T_{f} .
$$

The number of symbols allocated to the guard period $M_{g}$ can be written as:

$$
M_{g}=\frac{K \cdot T_{g}}{T_{s}} .
$$

Notice that special slots which provide a guard period are inserted in the conventional 5G NR and LTE frames when switching from downlink to uplink. On the other hand, switching from mini-slot uplink to downlink may not require a guard period due to the high processing capabilities of the $\mathrm{g}$ NodeB which will be switching from receiving to transmitting. It can be noticed that the proposed approach will generate interference from the LTE e-NodeB to the $5 \mathrm{G}$ g-NodeB, and from the 5G UE to the LTE UE during the mini-slot uplink transmission. This interference reduces the mini-slot efficiency and it may result in a complete loss of the mini-slot uplink transmission for some users. A simple solution is to mute the LTE transmission during the 5G NR mini-slot uplink. This guarantees interference-free mini-slot uplink transmission (from the e-NodeB to the g-NodeB perspective). However, the LTE muting reduces the available LTE time-domain resources and it requires modification to the incumbent LTE system.

To overcome these limitations, we propose keeping the LTE system unchanged (i.e., without resource muting) and performing blind retransmission for the $5 \mathrm{G}$ users whose uplink transmission in the mini-slot is completely blocked by the LTE eNodeB interference. Such blocking happens when the interference degrades the mini-slot uplink signal quality to become below the minimum level at which the signal can be correctly decoded, which in turns triggers a blind retransmission. When the user receives data from the g-NodeB, the UE decodes it and sends a feedback including acknowledgment (ACK) or negative acknowledgment (NACK), hybrid automatic repeat request (HARQ), and channel quality indicator (CQI). This feedback is sent in the mini-slot uplink and is received by the g-NodeB which responds according to the feedback in the mini-slot uplink. In some cases, the g-NodeB may not be able to decode the feedback in the mini-slot uplink (e.g., due to the additional interference from the LTE eNodeB as mentioned above). In these cases, the g-NodeB assumes that the user feedback in the mini-slot uplink is NACK (i.e., the worst case scenario) and it retransmits the last packet with a coding/modulation scheme corresponding to the most recent correctly received CQI feedback. The flow chart in Fig. 5 illustrates how and when the blind retransmission is triggered.

\section{PERformance Evaluation}

\section{A. Simulation Setup and Scenarios}

To evaluate performance of the proposed solution, detailed system-level simulations have been performed using the $5 \mathrm{G}$ Innovation Centre system-level simulator (5GIC-SLS). We consider the $5 \mathrm{G} \mathrm{NR}$ and the LTE to be deployed in the 3.4 $\mathrm{GHz}-3.8 \mathrm{GHz}$ band. The ECC deployment scenario of [6] has been adopted where the networks are shifted by $288 \mathrm{~m}$ with $90^{\circ}$ (best case) and by $70 \mathrm{~m}$ with $60^{\circ}$ (conservative 


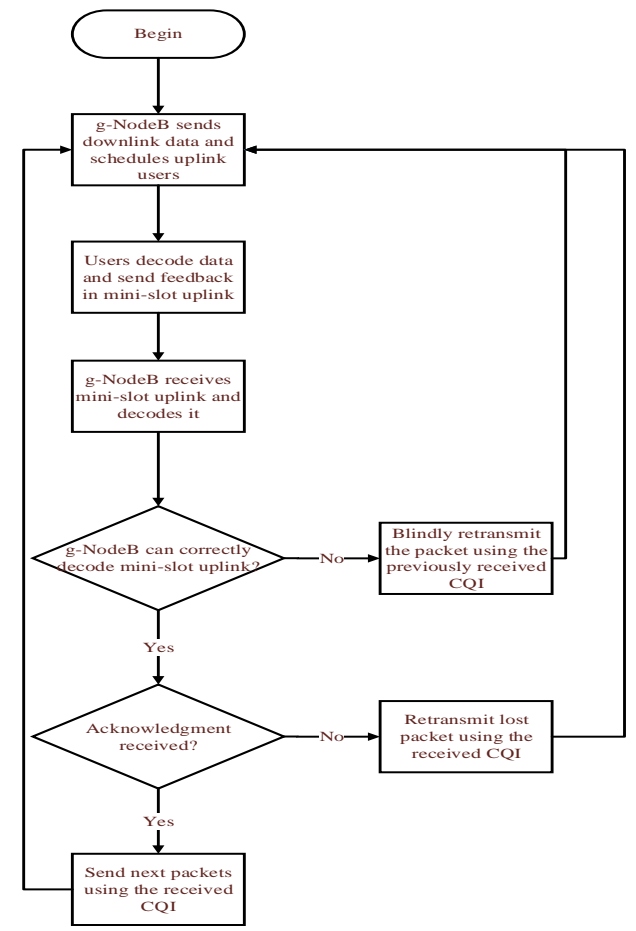

Figure 5: Blind retransmission flow chart Table I: Simulation parameters

\begin{tabular}{|c|c|}
\hline Parameter & Value \\
\hline Layout & $\begin{array}{l}\text { Hexagonal grid, wraparound } \\
1 \text { ring ( } 7 \text { sites) with } 3 \text { sectors per BS }\end{array}$ \\
\hline Inter-Site Distance (ISD) & $500 \mathrm{~m}$ \\
\hline BS antenna height & $25 \mathrm{~m}$ \\
\hline BS antenna model & $\begin{array}{l}\text { 5G NR: } 8 \times 8 \times 2 \text { elements, grid of beam } \\
\text { LTE: } 3 \text { GPP TR } 36.814 \text { model }\end{array}$ \\
\hline BS noise figure & $5 \mathrm{~dB}$ \\
\hline BS max transmitted power & $51 \mathrm{dBm}$ \\
\hline Shadowing & Correlated with 0.5 inter-site correlation \\
\hline Adjacent channel leakage ratio & $45 \mathrm{~dB}$ \\
\hline UE max transmitted power & $23 \mathrm{dBm}$ \\
\hline UE antenna type & Omni directional \\
\hline UE antenna gain & $-4 \mathrm{dBi}$ \\
\hline UE antenna height & $1.5 \mathrm{~m}$ \\
\hline UE noise figure & $9 \mathrm{~dB}$ \\
\hline Propagation models & $\begin{array}{l}\text { Macro BS } \rightarrow \text { Macro BS: Free space } \\
\text { Macro BS } \rightarrow \text { Macro UE: 3D UMa }\end{array}$ \\
\hline Subcarrier spacing & LTE: $15 \mathrm{kHz}, \mathrm{NR}: 30 \mathrm{kHz}$ \\
\hline
\end{tabular}

case). Both systems use a $10 \mathrm{MHz}$ simulation bandwidth with transmission mode 1 and proportional fair scheduler. $10 \mathrm{UE}$ per sector are deployed for each network. A frame latency threshold of $4 \mathrm{~ms}$ is assumed for the allocation of the mini-slot uplink. Other simulation parameters are provided in Table I.

The interference model considers both the intra-system interference and the inter-system interference. The former is the interference generated by users (or BSs) of the same system in the same resource block. In the $5 \mathrm{G}$ for example, the intrasystem interference in the downlink towards a certain $5 \mathrm{G}$ user is the summation of the interference from all non-serving $5 \mathrm{G}$ g-NodeBs transmitting in the same resource block(s) allocated to the affected $5 \mathrm{G}$ user, while the intra-system interference in the uplink towards a certain $5 \mathrm{G}$ g-NodeB is the summation of interference from all other $5 \mathrm{G}$ g-NodeB users transmitting in

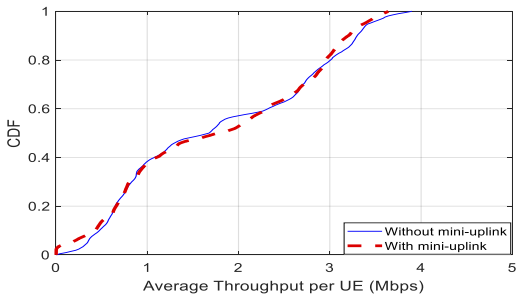

Figure 6: 5G NR UE throughput, best case scenario.

Table II: Throughput gain and loss of mini-slot uplink

\begin{tabular}{|c|c|c|}
\hline Scenario & Parameter & Results \\
\hline \multirow{2}{*}{ Best case } & Average throughput gain/loss & $-0.8 \%$ \\
\cline { 2 - 3 } & $5 \mathrm{G}$ users with lost mini-slot uplink & $1.9 \%$ \\
\hline \multirow{2}{*}{ Conservative case } & Average throughput gain/loss & $-2.3 \%$ \\
\cline { 2 - 3 } & $5 \mathrm{G}$ users with lost mini-slot uplink & $2.4 \%$ \\
\hline
\end{tabular}

the same resource block being evaluated (assuming the frames used by all BSs/users of the same system are synchronized). This interference reduces with respect to the distance from the BS because it is a function of the transmit power, antenna orientation, radiation pattern and gain as well as the path loss model which itself depends on the distance between the user and the BS. The inter-system interference is the interference from elements (i.e., users and/or BSs) of one system (e.g., the LTE) towards the other system (e.g., the 5G). In the synchronized parts of the frames, the inter-system interference in the downlink is the summation of interference from all BSs of one system towards the user of the other system. The inter-system interference in the uplink is the summation of interference from all users of one system towards BS of the other system. When the two systems are not fully synchronized (i.e., when the 5G NR mini-slot uplink is aligned with the LTE downlink), then the inter-system interference also includes the interference from BSs of one system (i.e., the LTE e-NodeBs) towards BSs of the other system (i.e., the 5G g-NodeB).

The inter-system interference is a function of the interfering node transmit power, the adjacent channel leakage ratio, the antenna orientation, radiation pattern and gain as well as the path loss model which itself depends on the distance between the user and the BS (or between the LTE and the 5G BSs in the unsynchronized case). Hence, the channel leakage interference reduces with respect to the distance from the BS. It is worth mentioning that we consider the 3-dimensional distance in the path loss model (and consequently in the interference model) by taking into account the antenna-to-antenna distance (i.e., by considering both the antenna height and the direct distance to incorporate the 3-dimensional distance in the interference model as well as in the received power model). The reader is referred to [6] and [14] for the detailed modelling of the received power, interference, 3 -dimensional distance and the path loss models.

\section{B. Simulation Results}

Figs. 6 and 7 show cumulative distribution function (CDF) of the 5G NR UE downlink throughput with and without minislot uplink in the best case and the conservative deployment scenarios, respectively. Table II shows the gain/loss in average UE throughout in the $5 \mathrm{G} \mathrm{NR}$ with mini-slot uplink w.r.t. the $5 \mathrm{G}$ NR without mini-slot uplink, along with the percentage of users with a complete loss of the mini-slot uplink. A throughout loss of $2.3 \%$ can be observed when employing the $5 \mathrm{G}$ NR frame with mini-slot uplink is observed. This can be 


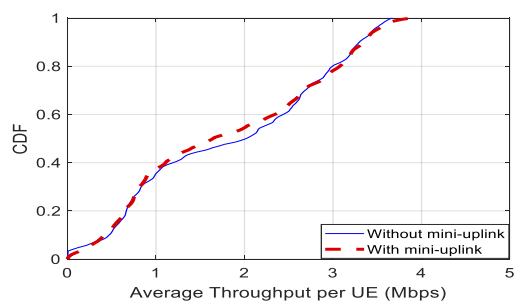

Figure 7: 5G NR UE throughput, conservative scenario.

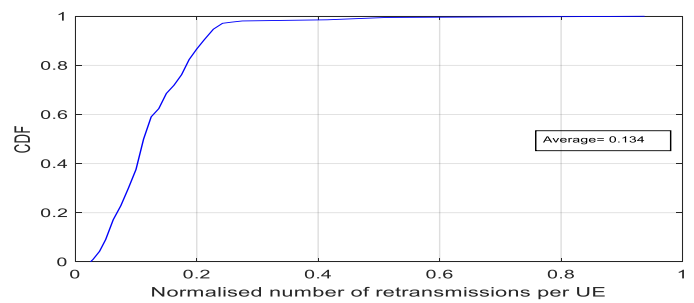

Figure 8: Retransmissions per UE, best case scenario.

traced to the fact that the mini-slot uplink reduces the number of symbols available for $5 \mathrm{G}$ NR downlink transmission. However, it allows more frequent uplink feedback, which in turn enables more updated scheduling decisions, CQI selection and less retransmissions. The net gain being negative indicates that the throughput loss due to the former slightly outweighs the throughput gain obtained by the latter.

In addition, Table II shows that $1.9 \%$ and $2.4 \%$ of $5 \mathrm{G}$ users cannot use the mini-slot uplink in the best case scenario and the conservative scenario, respectively. The g-NodeB performs blind retransmission for these users by using the most recent correctly received CQI feedback, which will contribute to the small reduction in the downlink throughput observed in Figs. 6 and 7. However, the mini-slot uplink can result in a significant reduction in the feedback and retransmission latency. For example, Fig. 8 shows the number of retransmissions per UE normalized with the total downlink simulation time. As can be noticed in Fig. 8, on average $13.4 \%$ of the total transmissions are retransmissions, and hence reducing the latency for these retransmissions will have a noticeable effect.

Table III shows the latency for ACK/HARQ and $1^{\text {st }}$ retransmissions in four scenarios with $0.5 \mathrm{~ms}, 1 \mathrm{~ms}, 2 \mathrm{~ms}$ and $4 \mathrm{~ms}$ processing delays. The mini-slot uplink reduces the $5 \mathrm{G}$ NR maximum HARQ latency by $25 \%, 25 \%, 33.3 \%$ and $25 \%$, and it reduces the maximum $1^{\text {st }}$ retransmission latency by $20 \%$, $20 \%, 25 \%$ and $16.7 \%$ with $0.5 \mathrm{~ms}, 1 \mathrm{~ms}, 2 \mathrm{~ms}$ and $4 \mathrm{~ms}$ processing delays, respectively. In addition, it guarantees the $5 \mathrm{G}$ eMBB $4 \mathrm{~ms}$ target for up to the $1^{\text {st }}$ retransmission with processing delays $\leq 1 \mathrm{~ms}$. Thus it can be said that the minislot uplink with blind retransmission enables coexistence of the latency-constrained 5G NR with the LTE-TDD.

\section{CONCLUSION}

In this paper, we investigated coexistence of the $5 \mathrm{G}$ NR and the LTE-TDD in sub-6 GHz bands. synchronized operation with fully aligned frames has been considered as the most effective solution to avoid the interference between the LTE e-NodeBs and the 5G g-NodeBs. Despite the potential gains, this approach comes at the expense of violating the $5 \mathrm{G}$ NR latency requirements. In this regard, we proposed to use minislot uplink signaling with blind retransmission to reduce the
Table III: Minimum (Min), maximum (Max) and average (Avg) latency in 5G NR with and without mini-slot uplink.

\begin{tabular}{|c|c|c|c|c|c|c|c|}
\hline \multirow{2}{*}{ Processing } & \multirow{2}{*}{ Delay Parameter } & \multicolumn{3}{|c|}{ Without Mini-Slot } & \multicolumn{3}{c|}{ With Mini-Slot } \\
\cline { 3 - 8 } & & Min & Max & Avg & Min & Max & Avg \\
\hline \multirow{2}{*}{$0.5 \mathrm{~ms}$} & Feedback (ms) & 1 & 4 & 2.1 & 1 & 3 & 1.6 \\
\cline { 2 - 8 } & Retransmission (ms) & 2 & 5 & 3.3 & 2 & 4 & 2.7 \\
\hline \multirow{2}{*}{$1 \mathrm{~ms}$} & Feedback (ms) & 1 & 4 & 2.1 & 1 & 3 & 1.6 \\
\cline { 2 - 8 } & Retransmission (ms) & 2 & 5 & 3.3 & 2 & 4 & 2.7 \\
\hline \multirow{2}{*}{$2 \mathrm{~ms}$} & Feedback (ms) & 2 & 6 & 3.3 & 2 & 4 & 2.8 \\
\cline { 2 - 8 } & Retransmission (ms) & 4 & 8 & 5.6 & 4 & 6 & 4.9 \\
\hline \multirow{2}{*}{$4 \mathrm{~ms}$} & Feedback (ms) & 4 & 8 & 5.8 & 4 & 6 & 4.8 \\
\cline { 2 - 8 } & Retransmission (ms) & 8 & 12 & 10 & 8 & 10 & 9 \\
\hline
\end{tabular}

5G NR frame latency whilst coping with the short burst of additional interference in the mini-slot uplink. Comprehensive simulations using our system-level simulator have been carried out for the proposed solution and shown to offer a tangible cost-benefit in terms of lower frame latency. At a low cost of $<3 \%$ throughput loss in the $5 \mathrm{G} \mathrm{NR}$, we can achieve up to $33 \%$ reduction in peak frame latency for feedback and acknowledgment, and up to $25 \%$ reduction in the $1^{\text {st }}$ retransmission latency. In addition, the $5 \mathrm{G}$ eMBB $4 \mathrm{~ms}$ latency target can be met with the mini-slot uplink as long as the processing delay is $\leq 1 \mathrm{~ms}$. Although the proposed solution has been tested for a single numerology and eMBB use case, the same approach can be adapted to other 5G NR numerologies and use cases such as URLLC.

\section{ACKNOWLEDGMENT}

We would like to acknowledge the support of the University of Surrey 5GIC (http://www.surrey.ac.uk/5gic) members for this work. We thank Kevin Holley, Stephen Temple and Chris Friel from the 5GIC Standards Sub-Group (SSG) for the constructive comments.

\section{REFERENCES}

[1] ITU, "ITU-R M.2410-0 Minimum requirements related to technical performance for IMT-2020 radio interface(s)," Tech. Rep., 2017.

[2] S.-Y. Lien et al., "5G new radio: Waveform, frame structure, multiple access, and initial access," IEEE Communications Magazine, vol. 55, no. 6, pp. 64-71, 2017.

[3] A. Mohamed, O. Onireti, M. Imran, A. Imran, and R. Tafazolli, "Control-data separation architecture for cellular radio access networks: A survey and outlook," IEEE Communications Surveys and Tutorials, vol. 18, no. 1, pp. 446-465, Firstquarter 2016.

[4] 3GPP, "System Architecture for the 5G System (5GS); Stage 2," Tech. Spec., September 2019, 3GPP TS 23.501, Version 16.2.0, Release 16.

[5] L. Wan, Z. Guo, and X. Chen, "Enabling Efficient 5G NR and 4G LTE Coexistence," IEEE Wireless Communications, vol. 26, no. 1, 2019.

[6] ECC, "ECC Report 296: National synchronisation regulatory framework options in 3400-3800 MHz: a toolbox for coexistence of MFCNs in synchronised, unsynchronised and semi-synchronised operation in 34003800 MHz," Tech. Rep., March 2019.

[7] 3GPP, "NR Base Station (BS) radio transmission and reception," Tech. Spec., 3GPP TS 38.104, Version 16.1.0, Release 16, 2019.

[8] E. Hossain and M. Hasan, "5G cellular: key enabling technologies and research challenges," IEEE Instrumentation \& Measurement Magazine, vol. 18 , no. 3, pp. 11-21, 2015.

[9] B. Yu, H. Ishii, and L. Yang, "System level performance evaluation of dynamic TDD and interference coordination in enhanced local area architecture," in IEEE 77th Vehicular Technology Conference, June 2013.

[10] 3GPP, "Study on New Radio (NR) access technology," Tech. Rep., 3GPP TR 38.912, Version 15.0.0, Release 15, 2018.

[11] E. Lähetkangas, K. Pajukoski, E. Tiirola, G. Berardinelli, I. Harjula, and J. Vihriälä, "On the tdd subframe structure for beyond $4 \mathrm{~g}$ radio access network." in Future Network \& Mobile Summit, 2013, pp. 1-10.

[12] E. Laehetkangas et al. " "On the selection of guard period and cyclic prefix for beyond 4G TDD radio access network," in Proc. of 19th European Wireless Conference, April 2013.

[13] A. Mohamed, O. Onireti, Y. Qi, A. Imran, M. Imran, and R. Tafazolli, "Physical layer frame in signalling-data separation architecture: Overhead and performance evaluation," in Proc. of 20th European Wireless Conference, May 2014, pp. 820-825.

[14] 3GPP, "5G, Study on channel model for frequencies from 0.5 to 100 GHz,” Tech. Rep., 3GPP TR 38.901, Version 14.3.0, Release 14, 2018 